\title{
VEMP using a new low-frequency bone conduction transducer
}

This article was published in the following Dove Press journal:

Medical Devices: Evidence and Research

Bo Håkansson, ' Karl-Johan

Fredén Jansson,' Tomas

Tengstrand, ${ }^{2}$ Leif Johannsen, ${ }^{3}$ Måns Eeg-Olofsson, ${ }^{4}$ Cristina

Rigato,' Elisabeth Dahlström, ${ }^{4}$

Sabine Reinfeldt

\section{'Department of Electrical} Engineering, Chalmers University of Technology, Gothenburg, Sweden; ${ }^{2}$ Department of Audiology, Sahlgrenska Academy, University of Gothenburg, Gothenburg, Sweden; ${ }^{3}$ Ortofon A/S, Nakskov, Denmark; ${ }^{4}$ Department of Otolaryngology, Head and Neck Surgery, Sahlgrenska Academy, University of Gothenburg, Gothenburg, Sweden

\section{Video abstract}

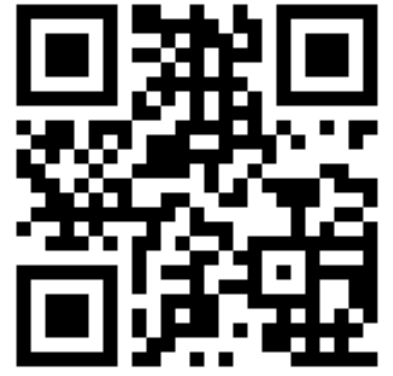

Point your SmartPhone at the code above. If you have a QR code reader the video abstract will appear. Or use: http://youtu.be/grWnXgTP2vs

Correspondence: Bo Håkansson Department of Electrical Engineering, Unit of Biomedical Signals and Systems, Chalmers University of Technology, Gothenburg, 4I2 96, Sweden

$\mathrm{Tel}+46317721807$

Email boh@chalmers.se
Objective: A new prototype bone conduction (BC) transducer B250, with an emphasized lowfrequency response, is evaluated in vestibular evoked myogenic potential (VEMP) investigations. The aim was to compare cervical (cVEMP) and ocular (oVEMP) responses using tone bursts at 250 and $500 \mathrm{~Hz}$ with $\mathrm{BC}$ stimulation using the B250 and the conventional B81 transducer and by using air conduction (AC) stimulation.

Methods: Three normal subjects were investigated in a pilot study. BC stimulation was applied to the mastoids in cVEMP, and both mastoid and forehead in oVEMP investigations.

Results: $\mathrm{BC}$ stimulation was found to reach VEMP thresholds at considerably lower hearing levels than in AC stimulation (30-40 dB lower oVEMP threshold at $250 \mathrm{~Hz}$ ). Three or more cVEMP and oVEMP responses at consecutive $5 \mathrm{~dB}$ increasing mastoid stimulation levels were only obtained in all subjects using the B250 transducer at $250 \mathrm{~Hz}$. Similar BC thresholds were obtained for both ipsilateral and contralateral mastoid stimulation. Forehead stimulation, if needed, may require a more powerful vibration output.

Conclusion: Viable VEMP responses can be obtained at a considerably lower hearing level with $\mathrm{BC}$ stimulation than by AC stimulation. The cVEMP and oVEMP responses were similar when measured on one side and with the B250 attached to both ipsilateral and contralateral mastoids. Keywords: vestibular investigation, air conduction, bone conduction, VEMP, cVEMP, oVEMP

\section{Introduction}

Bone conduction (BC) offers a well-researched parallel sound transmission path to the cochlea that is used in many applications. ${ }^{3,4}$ These applications range from consumer listening devices and hearing implants ${ }^{5-8}$ to audiometric testing devices. ${ }^{9,10}$ Our research group has now started to look into vestibular testing using $\mathrm{BC}$ stimuli, which is the focus of this paper.

In vestibular testing, the end organ for the bone-conducted vibrations is not the cochlear hair cells but instead the hair cells in the vestibular labyrinth, with the cupulas in the three orthogonal semicircular canals, and the otolith organ, comprising the essentially horizontal oriented macula utriculi and essentially the vertical oriented macula sacculi. ${ }^{11}$ A relatively new vestibular testing procedure named vestibular evoked myogenic potential (VEMP), has attracted increased interest in recent years. This technique, which was first clinically demonstrated by Colebatch and Halmagyi ${ }^{12}$ and Colebatch et al, ${ }^{13}$ consists of recordings of involuntary muscular response to stimulus from loud sound or skull vibration. In VEMP, a human body reflex activates muscular responses in order to restore posture and head position after unexpected rapid spatial movement. 
VEMP was originally measured by electromyography (EMG) recorded from electrodes on the skin over the sternocleidomastoideus muscle (SCM), also known as the cervical muscle (cVEMP). Later, EMG recordings from electrodes over the ocular inferior oblique orbital muscle (IOM) under the eye (oVEMP) were also introduced; for example, Todd et al. ${ }^{14} \mathrm{In}$ the first clinical studies, air-conducted (AC) sound was used for excitation, but later tapping or BC sound was also used; for example, Halmagyi et $a{ }^{15}$ developed a reflex hammer for forehead excitation.

Although not yet fully explored, there seems to be a consensus that cVEMP predominantly reflects saccular activation via the inferior vestibular nerve, ${ }^{16,17}$ whereas oVEMP reflects utricular activation via the superior vestibular nerve. ${ }^{18}$ There is scientific evidence that BC stimulation, similarly to AC stimulation, is evoking VEMP in guinea pigs. ${ }^{17,18}$ This finding opens for evoking VEMP responses with less sound burden than by $\mathrm{AC}$ and offers a possibility for patients with conductive hearing loss to undergo a VEMP investigation.

Detailed standardized methodologies for both cVEMP and oVEMP have been called for, but have yet to be established. In a review article, Papathanasiou et $\mathrm{al}^{20}$ presented guidelines regarding cVEMP, but stated that "we do not propose a single methodology as clinical use of cVEMPs is evolving and questions still exist about its underlying physiology and its measurement". The first challenge is to decide which stimulation modality should be used ( $\mathrm{AC}$ or $\mathrm{BC}$ ) and, if $\mathrm{BC}$ is chosen, which type of transducer should be used for the excitation. The second challenge is to decide which signal is the most efficient for obtaining a VEMP response with a particular transducer. While it is not mentioned so often, there is also a question about the clinical applicability and robustness of the different methods. Applicability is a highly relevant issue if VEMP is going to be used as a standard clinical procedure since it should then be easy to perform and the sound level required should not be uncomfortably high. Several options including either AC or BC stimulation are available to solve these issues but every solution has its pros and cons.

Halmagyi et al ${ }^{15}$ used manually applied tapped mechanical stimuli to evoke VEMP, although this technique never went into clinical practice, most likely because that manual tapping was found to be difficult to control and lacked repeatability. To overcome these issues, an electromagnetic tapping device was developed by co-authors in Brantberg et al. ${ }^{21}$ As far as we are aware, this device was only used as a research tool, possibly because of the high-emitted sound as the patients and test personnel had to wear ear protection. A more standardized automatic skull-tapping setup was developed later based on the electrodynamic moving coil type of transducer (B\&K Minishaker 4810; Brüel and Kjær, Denmark). ${ }^{22}$

While AC sound administered via an earplug in the ear canal is fairly effective for evoking VEMP, sound levels as high as $130-145 \mathrm{~dB}$ peak sound pressure level reference $20 \mu \mathrm{Pa}$ (SPL) are reported to be needed for short clicks of $0.1 \mathrm{~ms}$ duration in order to evoke clinically useful cVEMP response. ${ }^{13,16,17}$ Such sound levels appear to be uncomfortably high and imply a risk of causing at least a temporary cochlear damage ${ }^{23}$ or even persistent hearing loss. ${ }^{24}$ In the present study, tone bursts have been used instead of click sounds as they have been shown to evoke a stronger response in VEMP using BC stimulation. ${ }^{25}$ The risk of hearing damage might also generally be less when using a burst sound rather than a click sound. ${ }^{26}$

Using BC stimulation, it remains a challenge to provide a sufficiently strong mechanical stimulation to be clinically used in a rather simple way. The conventional BC transducer Radioear B71, initially developed for audiometric hearing investigations, has been used in both human studies ${ }^{27}$ and in animal models. ${ }^{19,28}$ While the B71 can evoke cVEMP at 500 $\mathrm{Hz}$, it often fails at lower frequencies and in oVEMP due to low power output. ${ }^{29}$ As the B71 does suffer from both poor power output and from high distortion at low frequencies, it has been improved by a newer design, the Radioear B81, which is based on the "balanced electromagnetic separation transducer" principle invented by Håkansson, ${ }^{9}$ offering a higher force output and lower distortion. ${ }^{10}$ Previous VEMP studies have also proposed reducing the stimulation frequency in VEMP from 500 to $250 \mathrm{~Hz}$ and even down to $100 \mathrm{~Hz}$ with the reservation that, at the lowest frequencies, response peaks without vestibular origin may show up..$^{30,31}$

It has been shown that the Minishaker B\&K 4810 is capable of evoking oVEMP at the forehead down to 100 $\mathrm{Hz},{ }^{18,22,30,31}$ but the drawback is its size and the fact that it must be held and attached manually. It should be noted that the Minishaker weighs $\sim 1.1 \mathrm{~kg}$, which is almost 30 times more than the B250 and the B81. Due to this reason, it will be difficult to apply the Minishaker with a stand-alone steel spring arrangement.

A new transducer element has now been developed, which is based on the B81 design, but is currently only available as a prototype called the B250. This device is slightly larger than the B81 and has a lower resonance frequency, placed at $250 \mathrm{~Hz}$ instead of $450 \mathrm{~Hz}$, as seen in Figure 1A and B. The considerably larger Minishaker B\&K 4810, which is based on the moving coil principle allowing larger deflections, is also included for comparative purposes (Figure 1). 
A

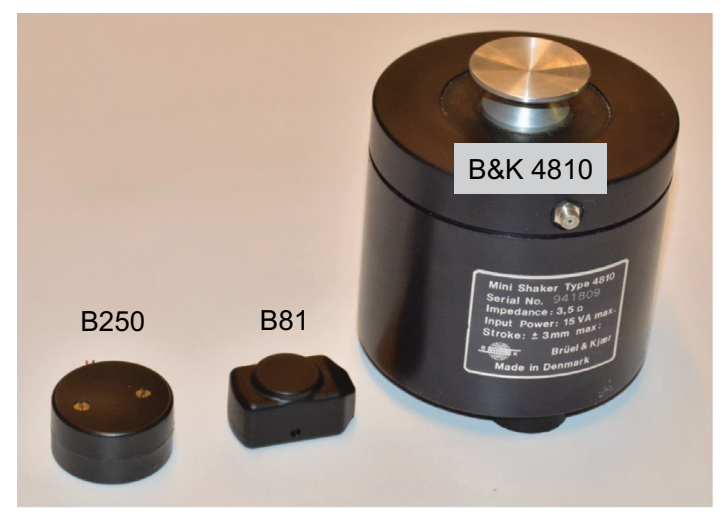

B

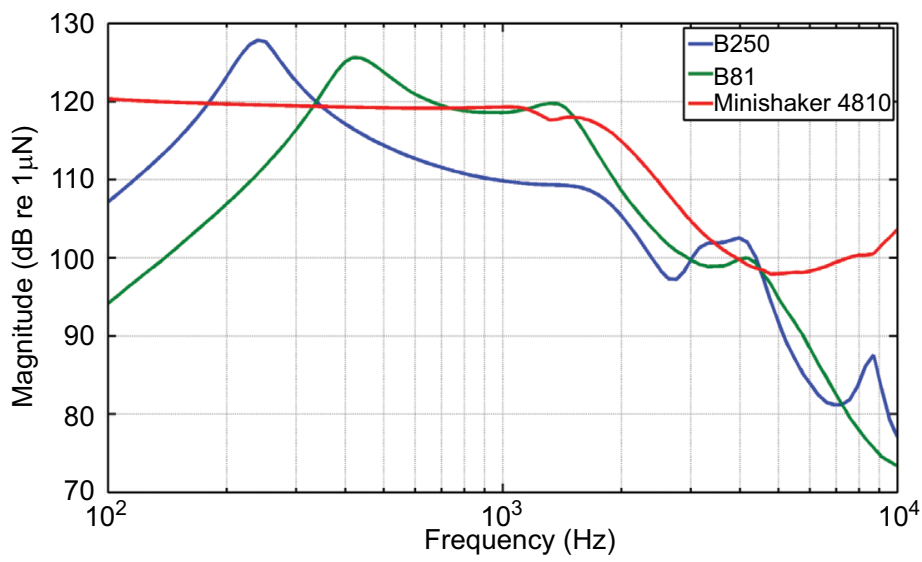

Figure I (A) B250 and B8I vibration transducers as well as the B\&K Minishaker 4810 (shown for comparative purposes) with their relative real size shown to the left and with their frequency responses to the right. (B) The frequency responses are shown as the output force level in $\mathrm{dB}$ re I $\mu \mathrm{N}$ measured on an artificial mastoid B\&K 4930 when driven by a sinusoidal signal with an amplitude of I Vrms from 100 to $10 \mathrm{k} \mathrm{Hz}$.

Notes: A static attachment force of $10 \mathrm{~N}$ between the transducer and the artificial mastoid was used.

Obviously, there are already several stimulation options for providing $\mathrm{BC}$ stimuli as an alternative to $\mathrm{AC}$, but it would be desirable if one and the same method could be used in both cVEMP and oVEMP. This is one of the reasons why the $\mathrm{B} 250$ transducer is proposed for VEMP investigations where the design is optimized for $250 \mathrm{~Hz}$ chosen as a compromise between size, output power, and desired stimulation frequency. In addition, this transducer has been designed with an electrical input impedance that is compatible with most commonly used hardware for vestibular testing.

This pilot study investigates the stimulation hearing level required to evoke robust VEMP responses from $\mathrm{AC}$ and $\mathrm{BC}$ at different stimulation attachments. Potential differences in the clinical interpretation of the VEMP responses, such as variability of asymmetry ratios, inhibitory/excitatory effects, vestibular source of the reflex, and vestibular labyrinth disorders, will not be taken into account. The clinical value of using the B250 will be investigated in a future study that is currently in the planning phase.

The overall aim of this pilot study was to measure cVEMP and oVEMP responses, evoked by 250 and $500 \mathrm{~Hz}$ tone bursts at different stimulation levels, using two miniaturized BC transducers (B250 and Radioear B81) applied to the mastoid and the forehead, and using AC stimulation.

The results will be analyzed and evaluated in view of the following research questions:

1. Is there any difference in stimulation hearing level required to evoke VEMP with $\mathrm{BC}$ and $\mathrm{AC}$ stimuli?

2. Can at least three typical biphasic VEMP responses be obtained at increasing stimuli levels with present BC transducers and available output levels?
3. Is there any difference between ipsilateral and contralateral stimulation level using $\mathrm{BC}$ at the mastoid?

\section{Materials and methods}

This is a pilot study based on three subjects: S1, 64 years (male); S2, 29 years (male); and S3, 28 years (female). All subjects are working in the hearing research group at Chalmers University of Technology. None of the subjects has had a known history of chronic ear disease or vestibular disorder. Placement of electrodes and bone conducting transducers are shown in Figure 2. Conventional skin adhesive electrodes (Neotrode from ConMed, Utica, NY, USA) were attached after the skin surface was rubbed by Nuprep Skin Prep Gel (Weaver, Aurora, CO, USA). The skin impedance was checked and verified to be below $2 \mathrm{k} \Omega$ between each of the tests to confirm the stability of the contact between the electrodes and the skin.

In cVEMP, the subjects were asked to tension the SCM to get a better electrode contact with the muscle by leaning to the opposite side and slightly bending the head forward. In oVEMP, the subjects were asked to gaze straight forward but upward as much as possible. This is found to achieve the highest possible oVEMP response signals with a suggested explanation that the measuring electrode comes closer to the inferior oblique muscle and that there is an increase of tonic extraocular muscle activity. ${ }^{32}$ The EMG signal was monitored by visual power meter, and the subject was asked to consciously keep the level at the meter's "green range" throughout the session, which is assumed to be sufficiently high.

A static pressure of $\sim 10 \mathrm{~N}$ was applied by use of a standard audiometric steel spring arrangement with an extension (an adaptor adding $2 \mathrm{~cm}$ laterally of the B250 and B81 
housing to increase the spring force). Ten Newton is twice the value specified for audiometric $\mathrm{BC}$ testing and corresponds to a peak force of $140 \mathrm{~dB}$ re $1 \mu \mathrm{N}$. All generator signals and the signal processing of measured signal were provided by the Eclipse EP25 including the OtoAccess software with a VEMP module (Interacoustics A/S, Middelfart, Denmark). This software was recently updated (mid-2017) and now includes the possibility of using $250 \mathrm{~Hz}$ for the BC stimulation; previously, the lowest frequency was $500 \mathrm{~Hz}$.

Frequency responses of the B250 (prototype from Ortofon $\mathrm{A} / \mathrm{S}$ ) and the B81 are shown in Figure 1B. These frequency responses were measured with a driving rod with a surface diameter $\mathrm{D}=15 \mathrm{~mm}\left(175 \mathrm{~mm}^{2}\right)$ for the $\mathrm{B} 81$ and $\mathrm{D}=20 \mathrm{~mm}$ $\left(315 \mathrm{~mm}^{2}\right)$ for the $\mathrm{B} 250$, and the graphs represent the $\mathrm{dB}$ output force level (OFL) at a fixed input voltage (1 Vrms) swept from 100 to $10 \mathrm{kHz}$. In repeated measurements, the response curves (Figure 1B) were almost identical.
In this study, only the frequencies $500 \mathrm{~Hz}$ (optimized for the B71/81) and $250 \mathrm{~Hz}$ (optimized in the BC250) were used. The stimulation signals comprised four cycles (shown in Figure $3 \mathrm{~A}$ and $\mathrm{B}$ ), having a total duration of $16 \mathrm{~ms}$ (250 $\mathrm{Hz})$ and $8 \mathrm{~ms}(500 \mathrm{~Hz})$, respectively. In the signal processing, averaging of 300 sequences was performed and the stimulation artifact was reduced by using alternating polarity; that is, subtraction of 180 degrees out of phase complementary registration.

Output levels of the transducers were calibrated on an artificial mastoid B\&K 4930 in order to translate the stimulation to $\mathrm{dB}$ normalized hearing level (nHL). This measure is used instead of $\mathrm{dB}$ HL to observe that these tone bursts are relatively short and can be considered as transient rather than continuous.

The OFLs are referred to as the reference equivalent threshold vibratory force levels (RETVFL) available from
A

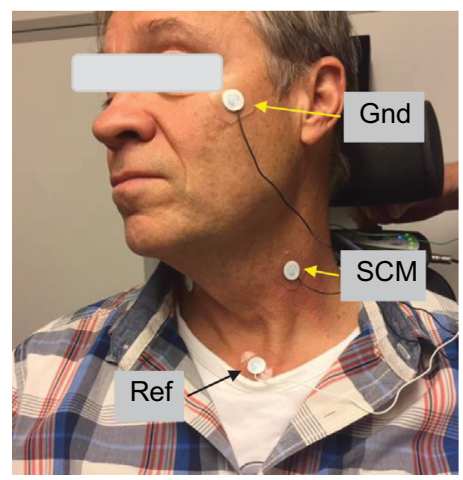

B

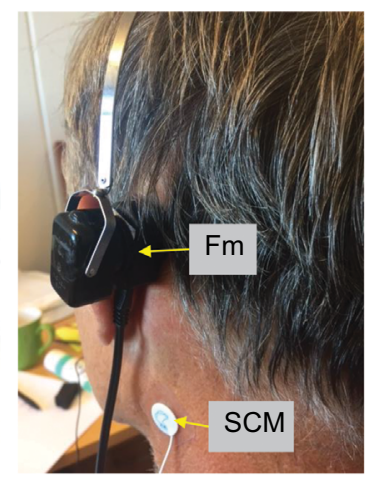

C

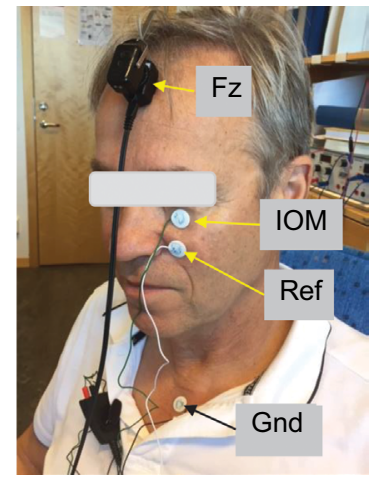

Figure 2 In cervical vestibular evoked myogenic potential (cVEMP), bipolar electrodes were placed: one at sternocleidomastoideus muscle (SCM) and the reference was placed at the upper rim of the sternum, and the ground at the upper cheek (A and B). In ocular vestibular evoked myogenic potential (oVEMP), bipolar electrodes were placed: one just beneath the eye over the inferior oblique muscle (IOM) and the reference $\sim 2 \mathrm{~cm}$ below, whereas the ground was placed at the upper rim of sternum (C). Bone-conducting transducers were applied using a steel spring arrangement either to the mastoid (Fm) just behind the pinnae at the virtual line through the ear canal opening and lateral eye hook or at the forehead $(\mathrm{Fz})$ on the midline just below the hairline (B and $\mathbf{C})$. Written informed consent has been obtained from the person in these images to publish.

Abbreviations: Fm, position of the vibration stimuli applied at the mastoid behind the pinnae; Fz, position of vibration stimuli applied at the midline of the skull just below the hairline; Gnd, ground electrode; Ref, reference electrode.
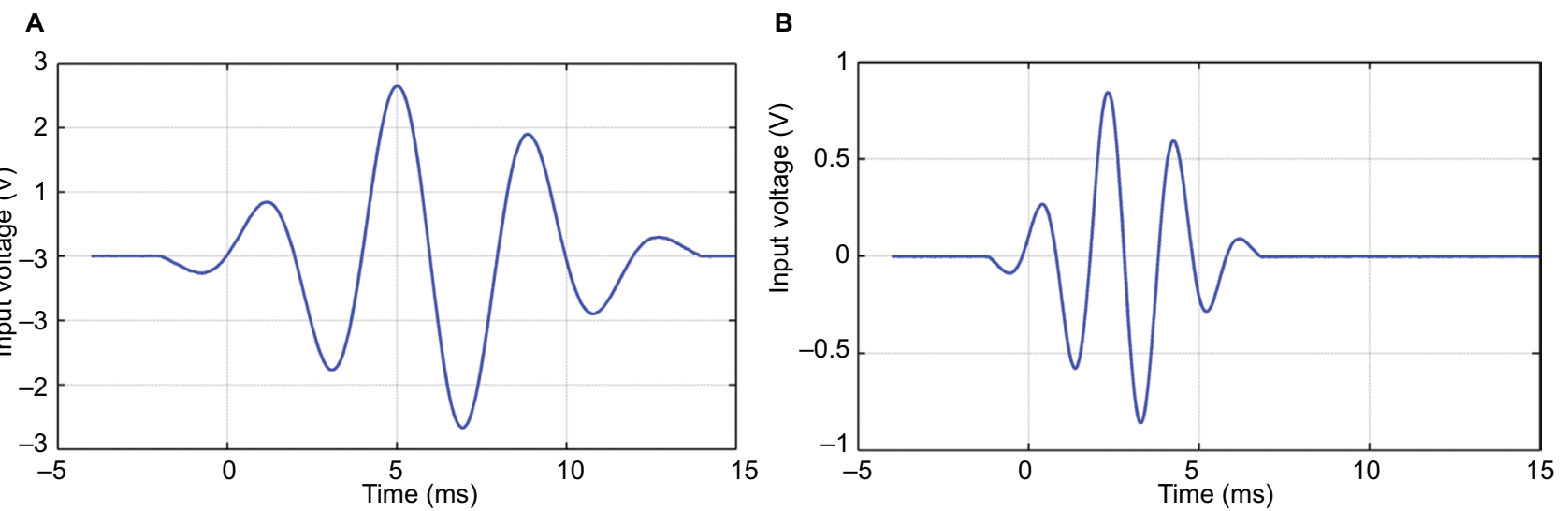

Figure 3 Graphs showing the burst stimuli used in the time domain (A) for $250 \mathrm{~Hz}$ and (B) $500 \mathrm{~Hz}$.

Notes: Ramping up is made during 1.5 cycles, plateau level is kept during one cycle, and fading down is made during 1.5 cycles. 
ISO $389-3^{1}$ and the electrical output from Eclipse was then referred to the $\mathrm{dB} n \mathrm{~nL}$ at which the VEMP responses were measured. At investigation frequencies (250 and 500 $\mathrm{Hz}$ ), the maximum force output level from the transducers, when driven at maximum output from the Eclipse hardware, was $75 \mathrm{~dB}$ nHL for B250 at both frequencies, whereas for $\mathrm{B} 81$, the maximum output was 65 and $85 \mathrm{~dB} \mathrm{nHL}$ for 250 and $500 \mathrm{~Hz}$, respectively. The same calibration was made for the AC sound where stimulation peak sound levels ( $\mathrm{dB}$ $\mathrm{nHL}$ ) are referred to the peak levels corresponding to the dB HL threshold levels, taken from ISO $389-2^{2}$ for insert earphones.

In AC and mastoid stimulation, a total of 72 measurements were made: three subjects, three stimulation methods (B250, B81, and AC), two frequencies $(250$ and $500 \mathrm{~Hz}$ ), cVEMP and oVEMP, ipsilateral and contralateral stimulation (in BC, the transducer was moved from one side to the other keeping the electrodes in the same place). In oVEMP, from the forehead midline/hairline Fz stimulation, a total of 12 measurements were made: three subjects, two devices (B81 and B250), two frequencies (250 and $500 \mathrm{~Hz}$ ).

The graphs in Figure 4 are shown in order to define the biphasic characteristics of cVEMP and oVEMP responses in the present study. In cVEMP there is a first negative peak ("p1") followed by a positive peak ("n1") occurring at latencies of $\sim 13 \mathrm{~ms}$ ("p13") and $23 \mathrm{~ms}$ ("n23") using click stimulus. In this example, the latencies are rather at 22 and $32 \mathrm{~ms}$, respectively, so obviously "p13" and " 13 " should only be regarded as names not latencies. In oVEMP, there is instead a first upward peak (n1) and a subsequent negative peak (p1) occurring at a latency of $\sim 10 \mathrm{~ms}$ (“" 10 ”) and $15 \mathrm{~ms}$ ("p15") using click stimulus. In this example, the latencies are rather in the range of 23-26 and 30-32 ms, respectively. These latencies may vary considerably depending on stimuli levels, if the stimuli is provided by $\mathrm{AC}$ or $\mathrm{BC}$, or if click or burst signals are used; therefore, they are just named as $n 1$ and $\mathrm{p} 1$ in this paper.

VEMP threshold levels were determined with the descending technique; that is, starting with the highest sound level and then decreased in $5 \mathrm{~dB}$ steps. The VEMP threshold is defined as the stimulus level in $\mathrm{dB}$ nHL where the $\mathrm{n} 1-\mathrm{p} 1$ response has a peak-to-peak amplitude, at roughly expected latencies, that was at least two times larger than the overall noise variability in the signal. The noise peak-to-peak variability was $\sim 10 \mu \mathrm{V}$ in cVEMP and $1 \mu \mathrm{V}$ in oVEMP responses, which is actually the scale division in Figure 4. A "viable" VEMP response is defined as that, at least three consecutive increasing stimulus amplitudes ( $5 \mathrm{~dB}$ steps) result in similar or increasing biphasic VEMP response amplitudes. Repeated measurements, with weeks in between, showed that the VEMP thresholds were very stable and the thresholds were found to be within $\pm 5 \mathrm{~dB}$ which indicates a variability which is less than that in hearing threshold testing.

It is clear from Figure 4 that the cVEMP response amplitudes are considerably higher than the oVEMP response by the different scales (10 vs $1 \mu \mathrm{V})$, and that the oVEMP responses are noisier. Generally, oVEMP responses are therefore more affected by stimuli artifacts. If the burst stimulus is too long, these artifacts may interfere with the first peak (n1) in the oVEMP response, and it was found that the $16 \mathrm{~ms}$ for

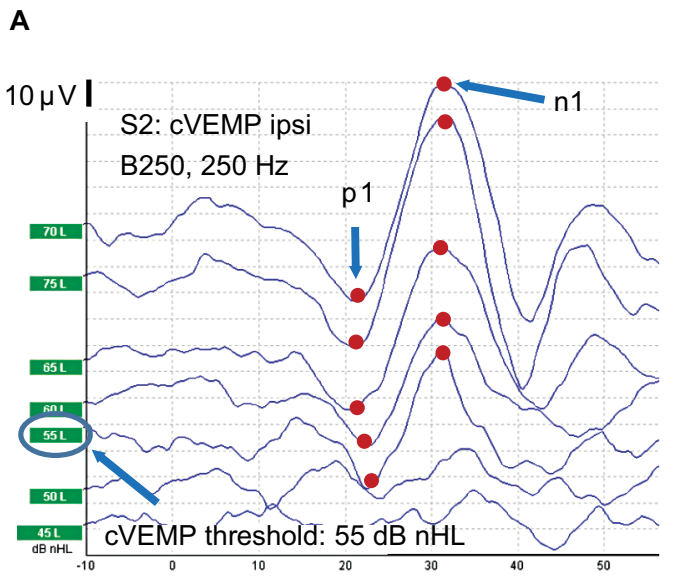

B

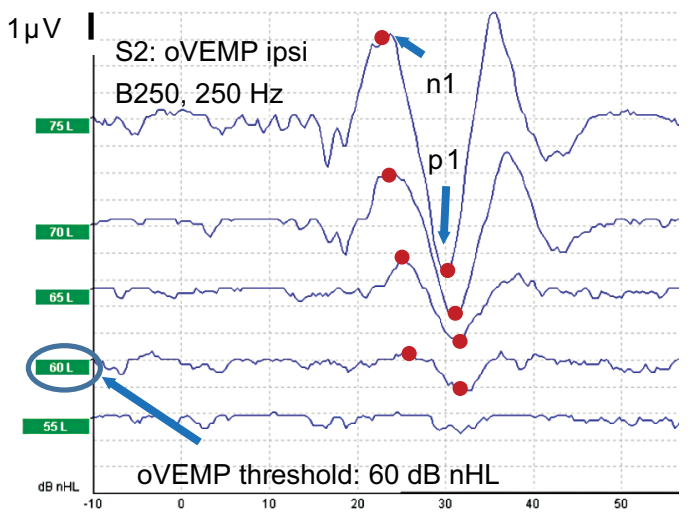

Figure 4 Typical clinically viable cervical vestibular evoked myogenic potential (cVEMP) (A) and ocular vestibular evoked myogenic potential (oVEMP) (B) responses from ipsilateral (ipsi) side using $250 \mathrm{~Hz}$ burst stimulation with B250 in subject S2.

Notes: The stimuli artifact is substantially reduced by a cancelation technique using alternated stimulation polarity and was normally not even seen in the responses, except for using B8I in oVEMP.

Abbreviation: $\mathrm{nHL}$, normalized hearing level. 
the $250 \mathrm{~Hz}$ burst was just on the limit of such interference using the B81 with Fz stimuli.

The study was conducted in accordance with the principles stated in the Declaration of Helsinki where applicable, and the test subjects, who are all research personnel in the field of BC, signed a written informed consent before entering the study. Participation in the study was voluntary and no remuneration was given to the test subjects. As this is a pilot study in a developmental phase, no formal ethical approval is required in Sweden (https://www.researchethics.lu.se/research-ethics-information/ethical-review/ when-is-ethical-permission-required).

\section{Results}

The left and center columns of Figure 5 show typical cVEMP and oVEMP responses from mastoid stimulation (Fm) in two subjects (S1 and S3) in order to illustrate the difference in hearing level required to obtain viable VEMP responses using B250 and AC at $250 \mathrm{~Hz}$. These results show that the B250 with the stimulus applied at the mastoid can evoke viable cVEMP and oVEMP responses at $250 \mathrm{~Hz}$, at a lower hearing level than in $\mathrm{AC}$ (in these examples 30-40 dB lower). It can also be seen in the upper-center and upper-right panels of Figure 5 that the oVEMP responses from $\mathrm{BC}$ stimulation resulted in the same threshold level with both ipsilateral and contralateral stimulation (within $2 \mathrm{~dB}$ among all subjects comparing average cVEMP and oVEMP thresholds for B250 at $250 \mathrm{~Hz}$ in Table 1). This indicates that attenuation of $\mathrm{BC}$ sound between the left and right vestibular organs is close to $0 \mathrm{~dB}$ for $250 \mathrm{~Hz}$. Viable VEMP responses were also seen in responses at $500 \mathrm{~Hz}$, but with lower peak-to-peak response amplitude levels for both the B250 and the B81.

All VEMP responses were similar in shape to those shown in Figure 5, and they are summarized in Table 1 by the threshold values in $\mathrm{dB} \mathrm{nHL}$. The average lowering in hearing levels using B250 versus AC stimulation among all subjects in this study was $39 \mathrm{~dB}$ for oVEMP and $32 \mathrm{~dB}$ for cVEMP, calculated from the average values in the right most column of Table 1. Altogether, these results indicate that cVEMP and oVEMP thresholds are generally reached at a considerably lower $\mathrm{dB}$ $\mathrm{nHL}$ stimulation for BC versus AC stimuli at $250 \mathrm{~Hz}$. At 500 $\mathrm{Hz}$, this difference is smaller, and the oVEMP threshold is on average $\sim 26 \mathrm{~dB}$ lower with B250 than with AC.

In Table 1, those conditions that were found viable (three biphasic VEMP responses with same or increasing amplitude levels) in all subjects are marked in green. Obviously, only the $\mathrm{B} 250$ at $250 \mathrm{~Hz}$ could obtain viable responses in all subjects for both cVEMP and oVEMP, whereas AC could do that in
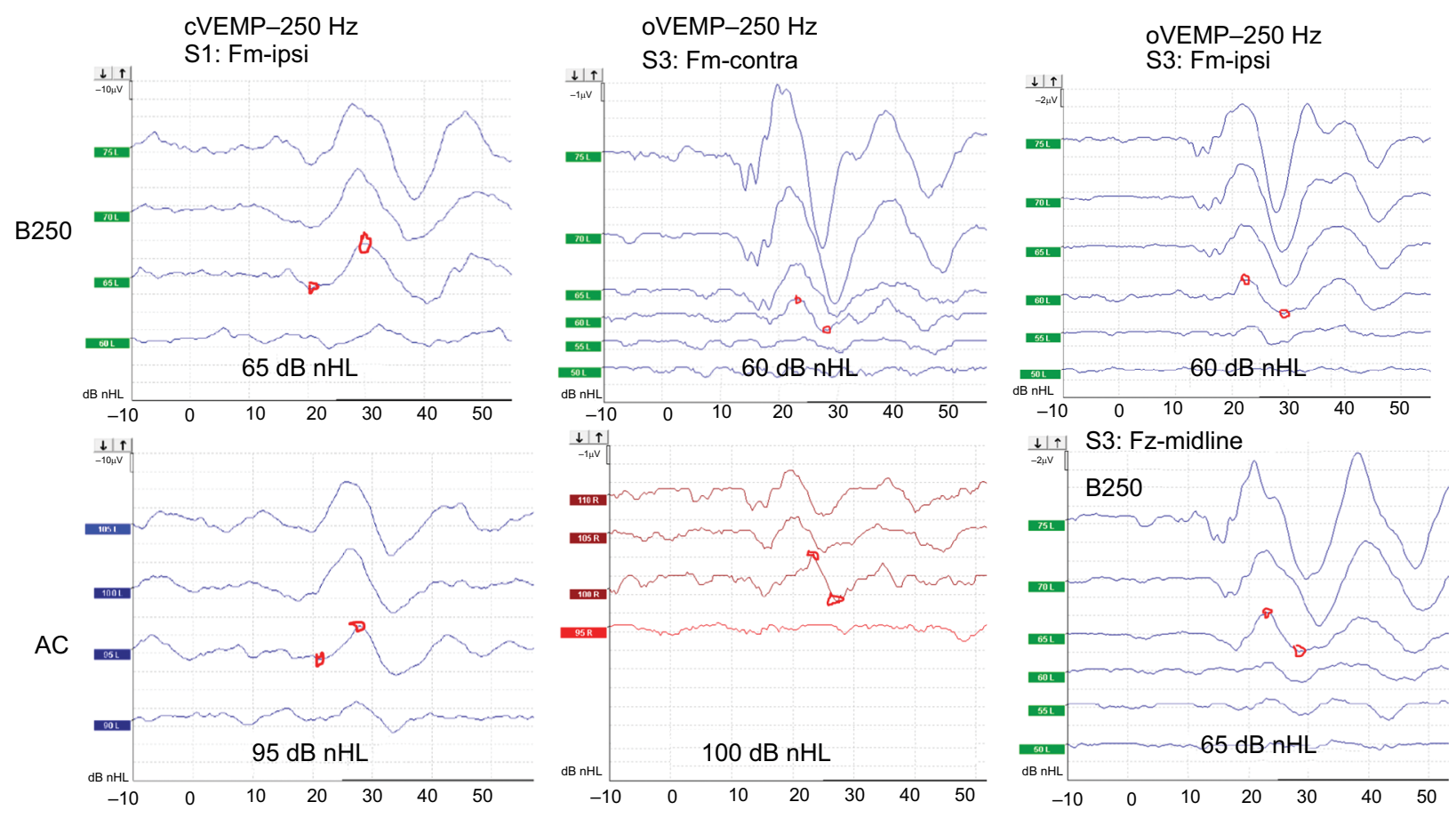

Figure 5 Typical VEMP responses where the separate curves are labeled by the $\mathrm{dB} \mathrm{nHL}$.

Notes: The responses with the lowest stimulation level with an expected p-n shape are noted in the boxes as the VEMP threshold level. Amplitude scales are different, cVEMP $(10 \mu \mathrm{V} /$ div) and oVEMP $(\mathrm{I}-2 \mu \mathrm{V} / \mathrm{div})$.

Abbreviations: cVEMP, cervical vestibular evoked myogenic potential; oVEMP, ocular vestibular evoked myogenic potential; $\mathrm{nHL}$, normalized hearing level; Fz, position of vibration stimuli applied at the midline of the skull just below the hairline; Fm, position of the vibration stimuli applied at the mastoid behind the pinnae; ipsi, ipsilateral; contra, contralateral. 
Table I VEMP thresholds - AC and mastoid stimuli $(\mathrm{dB} \mathrm{nHL})$

\begin{tabular}{|c|c|c|c|c|c|c|c|}
\hline \multirow{2}{*}{$\frac{\mathrm{Hz}}{250}$} & \multirow{2}{*}{$\begin{array}{l}\text { Stimulation } \\
\text { AC }\end{array}$} & \multirow{2}{*}{$\begin{array}{l}\text { VEMP } \\
\text { cVEMP: ipsi }\end{array}$} & \multirow{2}{*}{$\begin{array}{l}\mathbf{S I} \\
95\end{array}$} & \multirow{2}{*}{$\begin{array}{l}\mathbf{S 2} \\
75\end{array}$} & \multirow{2}{*}{$\begin{array}{l}\mathbf{S 3} \\
100\end{array}$} & \multicolumn{2}{|c|}{ Average } \\
\hline & & & & & & 90 & \multirow{6}{*}{$32 \mathrm{~dB}$} \\
\hline & \multirow[t]{3}{*}{ Max $110 \mathrm{~dB} \mathrm{HL}$} & contra & $>110$ & $>110$ & $>110$ & $>110$ & \\
\hline & & oVEMP: ipsi & $>110$ & $>110$ & 100 & $>107$ & \\
\hline & & contra & 95 & 105 & 100 & $100^{-}$ & \\
\hline & B8I & cVEMP: ipsi & $>65$ & 50 & 50 & $>55$ & \\
\hline & \multirow[t]{3}{*}{ Max $65 \mathrm{~dB} \mathrm{HL}$} & contra & $>65$ & 50 & 50 & $>55$ & \\
\hline & & oVEMP: ipsi & $>65$ & 60 & 50 & $>58$ & \multirow[b]{2}{*}{ dB } \\
\hline & & contra & 65 & 55 & 50 & $57^{3}$ & \\
\hline & B250 & cVEMP: ipsi & 65 & 55 & 55 & 58 & \multirow{5}{*}{$\mid$} \\
\hline & \multirow[t]{3}{*}{ Max $75 \mathrm{~dB} \mathrm{HL}$} & contra & 65 & 55 & 50 & 57 & \\
\hline & & oVEMP: ipsi & 65 & 60 & 60 & 62 & \\
\hline & & contra & 60 & 60 & 60 & 60 & \\
\hline \multirow[t]{12}{*}{500} & AC & cVEMP: ipsi & 90 & 75 & 100 & 88 & \\
\hline & \multirow[t]{3}{*}{ Max $110 \mathrm{~dB} \mathrm{HL}$} & contra & $>110$ & 110 & 100 & \multicolumn{2}{|l|}{$>107$} \\
\hline & & oVEMP: ipsi & 100 & 85 & 100 & \multicolumn{2}{|l|}{95} \\
\hline & & contra & 95 & 95 & 95 & \multicolumn{2}{|l|}{95} \\
\hline & B8I & cVEMP: ipsi & 80 & 60 & 65 & \multicolumn{2}{|l|}{68} \\
\hline & \multirow[t]{3}{*}{ Max $85 \mathrm{~dB} \mathrm{HL}$} & contra & 85 & 70 & 70 & \multicolumn{2}{|l|}{75} \\
\hline & & oVemp: ipsi & 75 & 75 & 70 & \multicolumn{2}{|l|}{73} \\
\hline & & contra & $>85$ & 70 & 70 & \multicolumn{2}{|l|}{$>75$} \\
\hline & B250 & cVEMP: ipsi & $>75$ & 60 & 60 & \multicolumn{2}{|l|}{$>65$} \\
\hline & \multirow[t]{3}{*}{ Max $75 \mathrm{~dB} \mathrm{HL}$} & contra & $>75$ & 75 & 70 & \multicolumn{2}{|l|}{$>73$} \\
\hline & & oVEMP: ipsi & 70 & 70 & 65 & \multicolumn{2}{|l|}{68} \\
\hline & & contra & 75 & 70 & 65 & \multicolumn{2}{|l|}{70} \\
\hline
\end{tabular}

Notes: Green cells: Viable VEMP responses obtained from at least three increasing stimulus levels in all subjects.

Abbreviations: ipsi, ipsilateral electrodes; contra, contralateral; HL, hearing level; $\mathrm{nHL}$, normalized hearing level; VEMP, vestibular evoked myogenic potential; cVEMP, cervical vestibular evoked myogenic potential; oVEMP, ocular vestibular evoked myogenic potential; AC, air conduction.

one condition at $250 \mathrm{~Hz}$ (cVEMP with ipsilateral stimuli) and three conditions at $500 \mathrm{~Hz}$ (cVEMP with contralateral stimuli and oVEMP with both ipsilateral and contralateral stimuli). With the B81, a clinically viable response was only obtained for oVEMP with ipsilateral stimuli at $500 \mathrm{~Hz}$.

Comparing B81 and B250 in Table 1 reveals no dramatic differences in threshold levels for cVEMP at either 250 or $500 \mathrm{~Hz}$. This is expected as the main differences for these two $\mathrm{BC}$ transducers are in the maximum output capability (shown in Table 1 under the transducer name in the "Stimulation" column). This finding is also in line with the assumption that the threshold at a certain frequency is evoked by the same level of mechanical stimulation independently of the transducer type and the frequency responses in Figure 1B. The differences in VEMP thresholds between the two transducers are $<5 \mathrm{~dB}$, on average, and may be related to intra-subject variability as the stimulation amplitudes are provided in 5 $\mathrm{dB}$ steps. However, it was found in the calibration procedure that the B81 had considerably higher distortion at $250 \mathrm{~Hz}$ compared to the B250 when driven at the highest output level. This is because the resonance frequency is approximately twice the stimulation frequency in the B81, which means that the second harmonic component will be considerably more amplified than the fundamental. This may also explain why in cVEMP, B81 had a lower VEMP threshold at $250 \mathrm{~Hz}$ than at $500 \mathrm{~Hz}$, whereas the B250 did not show such a large difference between these two frequencies.

With B250, viable responses are obtained for all subjects at $250 \mathrm{~Hz}$ (green cells in Table 1); however, it fails at $500 \mathrm{~Hz}$, whereas the situation is reversed for the B81 (even though B81 has only one green condition at $500 \mathrm{~Hz}$ ). This is most likely due to the difference in resonance frequencies and higher maximum output of 85 versus $75 \mathrm{~dB}$ nHL in favor of the $\mathrm{B} 81$ at $500 \mathrm{~Hz}$ and the opposite 75 versus $65 \mathrm{~dB} \mathrm{nHL}$ in favor of the B250 at $250 \mathrm{~Hz}$. These limitations are set generically by the transducer design and the maximum output voltage from Eclipse. It also appears that the VEMP thresholds at $500 \mathrm{~Hz}$ (range $68-75 \mathrm{~dB} \mathrm{nHL}$ ) are notably higher than the corresponding thresholds at $250 \mathrm{~Hz}$ (range 57-60 dB nHL). In real physical terms, however, the thresholds at $500 \mathrm{~Hz}$ are more similar to those at $250 \mathrm{~Hz}$, just more annoying at 500 $\mathrm{Hz}$, as the RETVFL at $250 \mathrm{~Hz}$ is $67 \mathrm{~dB}$ re $1 \mu \mathrm{N}$ and at 500 $\mathrm{Hz}$ is $58 \mathrm{~dB}$ re $1 \mu \mathrm{N}$; that is, they differ by $9 \mathrm{~dB}$ in absolute level the other way around. 
The lower right panel of Figure 5 shows a viable oVEMP response from $\mathrm{Fz}$ midline forehead position for subject $\mathrm{S} 3$ using the B250 at $250 \mathrm{~Hz}$. Whereas the youngest subjects (S2 and S3) reached their thresholds from Fz in almost all conditions, the oldest subject (S1) did not reach threshold in any condition (Table 2). Obviously, if an oVEMP response from $\mathrm{Fz}$ is desired for any physiological reason, a transducer with stronger mechanical output is needed if viable responses should be obtained in all patents.

\section{Discussion}

\section{General discussion of present results}

This paper presents results obtained using a new and relatively small prototype $\mathrm{BC}$ transducer that has been optimized for being driven at $250 \mathrm{~Hz}$, and is hence called the B250. We have shown that the B250 has the potential to be used for both cVEMP and oVEMP measurements and is compatible with conventional commercially available vestibular testing equipment. We have also shown that a simple headband arrangement (standard steel spring with adaptor extension to increase the static force) can be used. This transducer attachment to the skull bone was more pleasant to use and easy to apply with the B250 than with the B81, most likely because it has a larger contact area $\left(315 \mathrm{vs} 175 \mathrm{~mm}^{2}\right)$ and because it is designed with a slight concave surface to better fit the mastoid bone. In this study, all stimulation levels are presented in $\mathrm{dB}$ $\mathrm{nHL}$ in order to compare the subjective experienced sound level; in view of that $\mathrm{AC}$ stimulation is known to require a relatively high stimulation level to evoke VEMP. In this pilot study, it was found that the VEMP threshold could be reached at significantly lower levels with $\mathrm{BC}$ stimuli than with AC stimuli - an average of $39 \mathrm{~dB}$ lower using a stimulation frequency of $250 \mathrm{~Hz}$ in oVEMP measurements. This is a remarkable improvement and it was much appreciated by the subjects in this study as all of them experienced that the AC stimulus was on the verge of being painful. Perhaps

Table 2 oVEMP thresholds - midline $\mathrm{Fz}(\mathrm{dB} \mathrm{nHL})$

\begin{tabular}{llllll}
\hline $\mathbf{H z}$ & Stimulation & SI & S2 & S3 & Average \\
\hline $\mathbf{2 5 0}$ & B8 I & $>65$ & $>65$ & 55 & $>62$ \\
& $\begin{array}{l}\text { Max 65 dB HL } \\
\text { B250 }\end{array}$ & $>75$ & 70 & 65 & $>70$ \\
& $\begin{array}{l}\text { Max 75 dB HL } \\
\mathbf{5 0 0}\end{array}$ & & & & \\
& B8 I & $>85$ & 75 & 65 & $>75$ \\
& Max 85 dB HL & & & & \\
& B250 & $>75$ & 75 & 65 & $>72$ \\
& Max 75 dB HL & & & & \\
\hline
\end{tabular}

Abbreviations: oVEMP, ocular vestibular evoked myogenic potential; $\mathrm{Fz}$, position of vibration stimuli applied at the midline of the skull just below the hairline; $\mathrm{nHL}$, normalized hearing level; $\mathrm{HL}$, hearing level. unsurprisingly, transmission via $\mathrm{AC}$ to the vestibular organ is not optimized by nature like natural hearing via $\mathrm{AC}$ to the cochlea. This could explain why BC evokes VEMP responses at considerably lower hearing levels than AC.

Apart from comfort and sound level aspects, another driving force for doing VEMP investigations by using $\mathrm{BC}$ stimulation is that patients who have a suspected or confirmed conductive hearing loss could also be investigated. This study indicates that the B250 offers such a possibility, but a future study on patients with conductive hearing loss has been planned for confirmation.

It was also seen that the B250 emitted less electromagnetic radiation than the $\mathrm{B} 81$ (which is indeed known to be better than the $\mathrm{B} 71$ in this respect). Less electromagnetic radiation originates from the balanced transducer principle and the more shielding inherent with the design of the B250. A reduction of the electromagnetic radiation artifacts is an advantage not only for VEMP but also for auditory brainstem response measurements which will be the subject of a future study.

In summary, this pilot study indicates that the B250 can take the VEMP investigations to a new level and possibly form the basis for a standardized procedure. First, however, present pilot results must be confirmed in extended clinical studies to verify normative data (thresholds, latencies, etc) on a larger group of normal subjects and to evaluate the diagnostic value in different patient groups. Interesting patient groups for future clinical studies are patients with unilateral vestibular dysfunction (such as vestibular Schwannoma patients) and patients with superior semicircular canal dehiscence.

\section{Other differences between $A C$ and $B C$ stimulation in VEMP investigations}

As mentioned above, there are several reasons why AC is not optimal in VEMP investigations, the most important are the high sound levels needed and the fact that VEMP cannot be used in patients with significant conductive hearing loss. These issues could be solved by a BC stimulus, which leads to the question of what else differs between these two modalities.

In this discussion, we disregard neuro-physiological aspects, such as from where in the vestibular organ the reflex originates and which neuro-muscular transmission branches are involved. We also assume that the cVEMP response is mainly affecting the SCM on the ipsilateral side, whereas the oVEMP is mainly caused by a crossover effect; that is, stimulations of the ipsilateral vestibular organ will give a response in the contralateral IOM. Figure 6 illustrates 


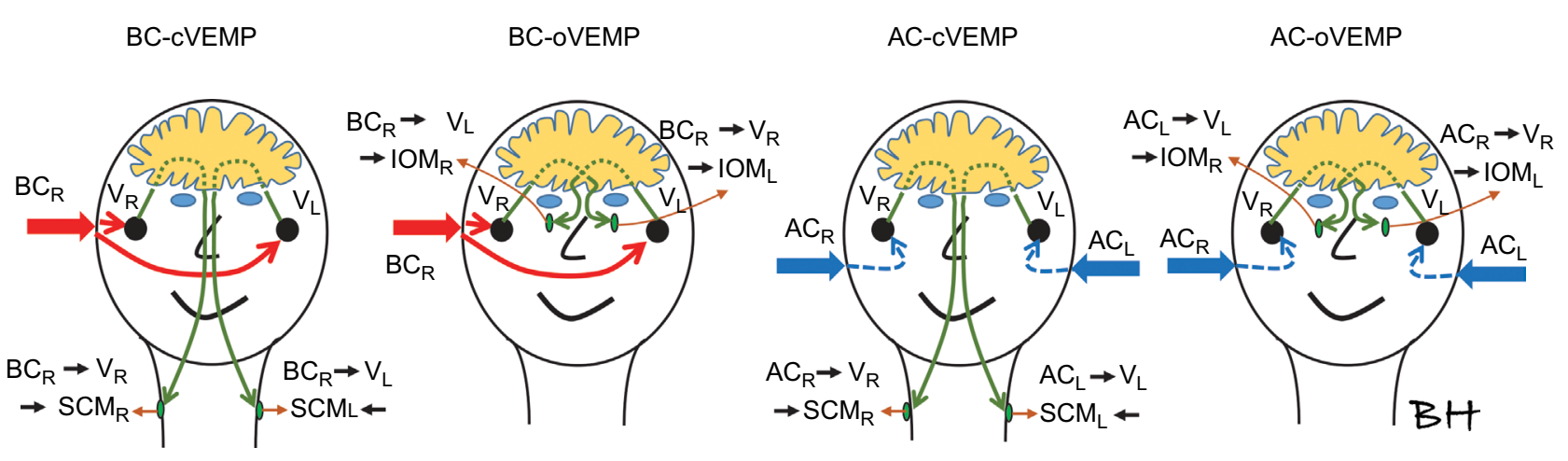

Figure 6 Using $B C$ stimuli, the vibration reaches the right $\left(V_{R}\right)$ and the left $\left(V_{L}\right)$ vestibular organ by approximately the same intensity at frequencies around $250 \mathrm{~Hz}$. Notes: Therefore, $B C$ stimuli only needs to be applied from one side $\left(B C_{R}\right)$, whereas with $A C$ stimuli it must be applied from both sides $\left(A C_{R}\right.$ and $\left.A C_{L}\right)$ in order to evoke CVEMP response in both cervical muscles $\left(S_{C} M_{R}\right.$ and $\left.S C M_{L}\right)$ or to evoke oVEMP in both orbital muscles (IOM ${ }_{R}$ and IOM $\left.I_{L}\right)$.

Abbreviations: AC, air conduction; BC, bone conduction; cVEMP, cervical vestibular evoked myogenic potential; IOM, inferior oblique orbital muscle; L, left; oVEMP, ocular vestibular evoked myogenic potential; R, right; SCM, sternocleidomastoideus muscle, $\mathrm{V}$, vibration.

these main vibration-evoked, neuro-muscular transmission branches for $\mathrm{AC}$ and $\mathrm{BC}$ stimulation.

It might be expected that using $\mathrm{AC}$ will involve some cross-talk to ipsilateral IOM in oVEMP as subject 3 had same threshold for both contralateral and ipsilateral stimulation at $250 \mathrm{~Hz}$, and all subjects had almost the same threshold at $500 \mathrm{~Hz}$ (this cross-talk is not shown in Figure 6). Without going into neurophysiological explanations, we just want to point out that this cross-talk effect from AC stimuli could also have $\mathrm{BC}$ origin. It is well known from audiology that an aerial sound in the ear canal and middle ear will cross over to the contralateral cochlea with a damping of $40-80 \mathrm{~dB},{ }^{33}$ depending on conditions. This is the reason why masking is needed in hearing threshold testing in patients with asymmetric hearing loss $>40 \mathrm{~dB}$. Therefore, it could also be that $\mathrm{AC}$ sound used in VEMP investigations can induce $\mathrm{BC}$ sound that is transmitted to the vestibular organ on the contralateral side, which in turn activates the ipsilateral IOM. The high acousto-mechanical transcranial damping $(40-80 \mathrm{~dB})$ can be compensated by a similar higher sensitivity for $\mathrm{BC}$ sound to evoke the reflex ( $39 \mathrm{~dB}$ in this study).

We also found that the attenuation between vestibular organs for BC was almost zero at $250 \mathrm{~Hz}$. Others have reported similar results in investigating the transcranial attenuation for $\mathrm{BC}$ sound between the cochleae and found it to be in the range of $0-5 \mathrm{~dB}$ at $250 \mathrm{~Hz} \cdot{ }^{34,35}$ This fact may simplify the BC-VEMP procedure, as the $\mathrm{BC}$ transducer only needs to be applied to one side, whereas in AC-VEMP the speaker must be applied to both sides. To include all four identified neuro-muscular reflex branches, electrodes must be applied to all four muscles involved for both $\mathrm{AC}$ and $\mathrm{BC}$ stimulation, as also shown in Figure 6.

From the discussion here, without considering detailed neurophysiological aspects, it is hard to see why VEMP using
BC stimuli should not have the same potential to reveal pathological differences in the neurological-myogenic pathways as from using AC stimuli. In addition, VEMP response levels in small children might be lower due to improper tension of SCM and less gazing upward to expose IOM, and thus BC-VEMP would give a better response signal, be more comfortable, and expose the child to less hearing hazard.

\section{Stimulation at the forehead, $\mathrm{Fz}$}

Using Fz stimulation instead of mastoid stimulation might raise some $\mathrm{BC}$ aspects that need to be considered. Below the free resonance frequencies of the human skull $(<\sim 800 \mathrm{~Hz})$, the skull behaves like a rigid body with the understanding that no external forces are connected. This was already pointed out by von Békésy ${ }^{36}$ and later confirmed by others; for example, Håkansson et $\mathrm{al}^{37}$ and Stenfelt and Goode. ${ }^{4}$ Sometimes this finding is mistakenly interpreted as meaning that all parts of the skull move like rigid body at low frequencies and also when a driving force is connected. When stimulated by an external force at lower frequencies, attached to the skull in a point like Fz or to the mastoids behind the ear, not only the skin but also the skull bone flexes at or near the attachment point. The forced response is different from a free response as it manifests itself by a dominant low-frequency anti-resonance in the mechanical impedance at the attachment point. ${ }^{37}$ This indicates that the skull, besides translational stiff solid body behavior, also has a compliance that changes/deforms the skull geometry already at low-frequency impacts. This also implies that the left and right vestibular labyrinths will move slightly differently when a low-frequency vibration or impact is applied to the mastoid, as compared to when the stimulation is applied to the Fz or the vertex.

Figure 7 shows that an inward stimulating force is applied to the midline $\mathrm{Fz}$ or the vertex, compressing the top portion 

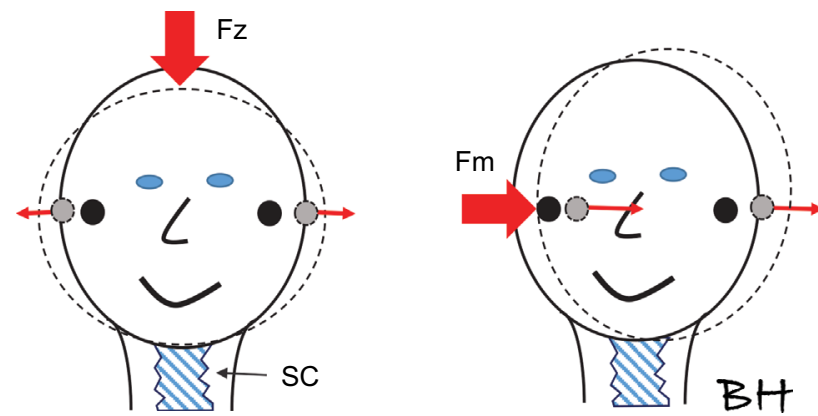

Figure $7 \mathrm{~A}$ simplistic view of how the skull deforms after a low-frequency impact from midline $\mathrm{Fz}$ (or vertex) and from the mastoid Fm.

Notes: It is important to consider that the skull rests on the spinal column (SC), which is quite stiff in the superior-inferior direction. The response movement/ acceleration of the vestibular labyrinth (solid circle in rest and gray circles after impact peak, assuming no delay between sides) will be out of phase with stimulation at $\mathrm{Fz}$ and mainly in phase with stimulation at Fm (impacts indicated by the wide red arrows and thin red arrows showing vestibular labyrinth motion direction). The acceleration response with stimulation at Fm may have a short time delay of $<\mathrm{l} \mathrm{ms}$ between vestibular motions ipsilateral versus contralateral side.

Abbreviations: Fz, position of vibration stimuli applied at the midline of the skull just below the hairline; Fm, position of the vibration stimuli applied at the mastoid behind the pinnae.

and expanding the middle portion, thus causing the vestibular labyrinths on both sides to move laterally at the same time. This also means that the vestibular labyrinths are stimulated with the same amplitude but asymmetrical 180 degrees out of phase (see red arrows in Figure 7A). On the other hand, if an external impact is applied to the mastoid (Fm), both vestibular labyrinths are moving in the same translational direction mainly in phase possibly with a short delay and minor rotational component. These phase differences between accelerations of the vestibular organs with Fz and Fm stimuli, respectively, were also confirmed by Westin and Brantberg, ${ }^{22}$ who also noted that the same force input level resulted in a five times higher lateral (interaural) acceleration if the stimulus was applied to the mastoids as compared to the vertex. This is understandable as a stimulation of the vestibular organ from the vertex or the Fz position relies mainly on the deformation of the superior part of the skull bone as the inferior part of the skull (the skull base) is relatively stiff in the superior-inferior direction due to the more or less rigid attachment to the stiff spinal column. In mastoid stimulation, on the other hand, the whole skull is fairly mobile in the lateral-medial direction, giving higher accelerations for a given stimulation force level Fm as compared to Fz, and includes both a deformation and a translational movement of the skull, as indicated in Figure 7.

It seems that VEMP responses from mastoid excitation are more natural in terms of how a postural position is normally restored after a lateral disturbance as developed by evolution. Events similar to Fz stimulation, caused by the out of phase motion of the vestibular organ, seem to be rare in real life and may thereby not evoke a strong response.
However, this does not mean that $\mathrm{Fz}$ or vertex stimulation does not have a special clinical value as some neural disorders may show up in Fz stimulation that is not shown in Fm (mastoid) stimulation, but we are not aware of any such cases. More clinical studies are needed before the Fz stimulation can be regarded as obsolete, but its potential exclusion and replacement by mastoid Fm stimulation would considerably simplify the procedures, as a bulky moving coil transducer like the B\&K 4810 would not be needed.

\section{Optimum stimulation frequency for VEMP investigations}

Another interesting question concerns the stimuli. We believe the impulsive short clicks should be avoided due to hazardous hearing risks and because they also suffer from low signalto-noise ratio in the response. In early VEMP investigations, burst signals were used with frequencies from $500 \mathrm{~Hz}$ and upwards. When BC-VEMP was introduced, it seemed that $500 \mathrm{~Hz}$ was the most popular frequency. This was most likely related to that resonance frequency of the Radioear B71, and later the B81, have their main resonance frequency in that frequency region. Lower stimulation frequencies were practically excluded due to the poor performance of these transducers below the resonance frequency. However, later studies using the B\&K 4810 Minishaker indicate that lower frequencies can be advantageous in oVEMP when sufficient vibration power is generated. The most effective frequency for utricular activation is reported to be as low as $100 \mathrm{~Hz}$, but still no consensus is available regarding the most optimal frequency. ${ }^{18,22}$

Stimulation at $100-125 \mathrm{~Hz}$ most likely requires a transducer of moving coil type in order to allow for larger deflections than in variable reluctance type transducers, such as those in B71 and B81 the maximum deflection of which is considerably lower and limited by relative small air gaps. ${ }^{10} \mathrm{In}$ the present study, a prototype transducer B250 was developed with a resonance frequency of $250 \mathrm{~Hz}$ optimized for $250 \mathrm{~Hz}$ stimulation. The $250 \mathrm{~Hz}$ resonance frequency was chosen as a compromise between the frequently used frequency of $500 \mathrm{~Hz}$ in cVEMP, suitable for the B71/81, and 100-125 Hz suitable for the B\&K 4810 Minishaker in oVEMP. Lowering the resonance frequency of the B250 even further is another viable option but that would require a considerably bigger transducer size, given that the resonance frequency is inversely proportional to the square root of the counter-weight mass. ${ }^{9}$ The choice of $250 \mathrm{~Hz}$ for the B250 was made not only because it allows for a reasonable size of the transducer but also because $250 \mathrm{~Hz}$ is one of the commonly used octave 
frequencies in audiometry where B71/B81 transducers have some limitations and where the B250 might be a better future alternative for audiometric testing.

\section{Conclusions}

In this pilot study, where the results are indicative, it was found that:

1. VEMP thresholds were reached at a considerably lower hearing level with $\mathrm{BC}$ stimulation than with $\mathrm{AC}$ stimulation - as much as 30-40 dB lower. Therefore, using $\mathrm{BC}$ stimulation in VEMP investigations may be more comfortable for the patient and expose the patient to a reduced hearing hazard.

2. cVEMP and oVEMP responses were evoked at three or more stimulation levels in all subjects, and all conditions were tested with a $250 \mathrm{~Hz}$ burst signal and using the prototype B250 transducer applied with a steel spring arrangement to one of the mastoids. It was noted that the B81 and AC stimuli performed better at $500 \mathrm{~Hz}$ than at $250 \mathrm{~Hz}$, although viable responses were not obtained in all subjects.

3. It was found that ipsilateral and contralateral stimulation levels using $\mathrm{BC}$ at the mastoid were almost the same at $250 \mathrm{~Hz}$. This indicates that both vestibular organs can be stimulated from only one of the mastoids in order to get viable cVEMP and oVEMP responses on both sides.

4. Forehead stimulation $\mathrm{Fz}$ did not evoke oVEMP response in all subjects with the B81/B250, possibly because of an unfavorable BC transmission to the vestibular organ. If Fz stimulation is deemed clinically necessary, this may necessitate a stronger device, such as B\&K 4810, driven at a frequency below $250 \mathrm{~Hz}$.

Using $\mathrm{BC}$, these results indicate that patients having a significant $\mathrm{BC}$ hearing loss can also be investigated with VEMP. We believe that the results in this pilot study indicate the potential of using BC in VEMP investigations; however, future clinical studies are needed in order to confirm these results and evaluate the clinical value.

\section{Acronyms and abbreviations}

$\mathrm{AC}$, air conduction; A weighting, filter shape similar to the response of the human ear at speech level sounds; B81/B71, commercially available audiometric bone conductors from InterAcoustics in Denmark; BC, bone conduction; BEST, balanced electromagnetic separation transducer; EMG, electromyography; Fm, position of the vibration stimuli applied at the mastoid behind the pinnae; Fz, position of vibration stimuli applied at the midline of the skull just below the hairline; IOM, inferior oblique muscle; LAeq, a weighted equivalent SPL over a period of time; OFL, output force level in $\mathrm{dB}$ relative to $1 \mu \mathrm{N}$; oVEMP, ocular vestibular evoked myogenic potential; RETSPL, reference equivalent threshold SPL according to ISO 389-2; RETVFL, reference equivalent threshold vibratory force levels according to ISO 389-3; ${ }^{1}$ SC, spinal column; SCM, sternocleidomastoideus muscle; SPL, sound pressure level in $\mathrm{dB}$ re $20 \mu \mathrm{Pa}$; VEMP, vestibular evoked myogenic potential; VR, VL, right and left vestibular organ; Vrms, volt root mean square; cVEMP, cervical vestibular evoked myogenic potential; $\mathrm{dB}$ HL, decibel hearing level relative to normal hearing threshold with continuous sinus signal; $\mathrm{dB} \mathrm{nHL}$, decibel normalized hearing level - peak level of transient signal relative to peak level of normal threshold with continuous sinus signal.

\section{Acknowledgment}

This study was supported by grants from Swedish Innovations Agency (Vinnova) and The Swedish Association of Hard of Hearing People (HRF). Equipment for this study was supported by Ortofon A/S and Interacoustics A/S, Denmark.

\section{Disclosure}

The corresponding author $(\mathrm{BH})$ holds patents regarding the BEST principle and LJ is an employee of Ortofon $\mathrm{A} / \mathrm{S}$, which manufactured the prototype bone conduction transducer B250 for this study. The authors report no other conflicts of interest in this work.

\section{References}

1. ISO 389-3. Reference zero for the calibration of audiometric equipment - Part 3: Reference equivalent threshold vibratory force levels for pure tones and bone vibrators. Geneva, Switzerland: International Organization; 2016. Available from: https://www.iso.org/standard/59759.html. Accessed August 28, 2018.

2. ISO 389-2. Acoustics - Reference zero for the calibration of audiometric equipment - Part 2: Reference equivalent threshold sound pressure levels for pure tones and insert earphones. Geneva, Switzerland: International Organization; 2016. Available from: https://www.iso.org/ standard/4378.html. Accessed August 28, 2018.

3. Eeg-Olofsson M, Stenfelt S, Taghavi H, et al. Transmission of bone conducted sound - correlation between hearing perception and cochlear vibration. Hear Res. 2013;306:11-20.

4. Stenfelt S, Goode RL. Transmission properties of bone conducted sound: measurements in cadaver heads. J Acoust Soc Am. 2005;118(4):2373-2391.

5. Håkansson B, Tjellström A, Rosenhall U, Carlsson P. The bone-anchored hearing aid. Principal design and a psychoacoustical evaluation. Acta Otolaryngol. 1985;100(3-4):229-239.

6. Eeg-Olofsson M, Håkansson B, Reinfeldt S, et al. The bone conduction implant - first implantation, surgical and audiologic aspects. Otol Neurotol. 2014;35(4):679-685.

7. Reinfeldt S, Taghavi H, Eeg-Olofsson M, Hakansson B. New developments in bone-conduction hearing implants: a review. Med Devices (Auckl). 2015;8:79-93. 
8. Taghavi H, Håkansson $\mathrm{B}$, Reinfeldt $\mathrm{S}$, et al. Technical design of a new bone conduction implant (BCI) system. Int J Audiol. 2015;54(10):736-744.

9. Håkansson BE. The balanced electromagnetic separation transducer a new bone conduction transducer. J Acoust Soc Am. 2003;113(2):818-825.

10. Jansson KJ, Håkansson B, Johannsen L, Tengstrand T. Electro-acoustic performance of the new bone vibrator Radioear B81: a comparison with the conventional Radioear B71. Int J Audiol. 2015;54(5):334-340.

11. Goldberg JM, Wilson VJ, Cullen KE. The Vestibular System - A Sixth Sense, Textbook. Oxford: Oxford University Press; 2012.

12. Colebatch JG, Halmagyi GM. Vestibular evoked potentials in human neck muscles before and after unilateral vestibular deafferentation. Neurology. 1992;42(8):1635-1636.

13. Colebatch JG, Halmagyi GM, Skuse NF. Myogenic potentials generated by a click-evoked vestibulocollic reflex. J Neurol Neurosurg Psychiatry. 1994;57(2):190-197.

14. Todd NP, Rosengren SM, Aw ST, Colebatch JG. Ocular vestibular evoked myogenic potentials (OVEMPs) produced by air- and boneconducted sound. Clin Neurophysiol. 2007;118(2):381-390.

15. Halmagyi GM, Yavor RA, Colebatch JG. Tapping the head activates the vestibular system: a new use for the clinical reflex hammer. Neurology. 1995;45(10):1927-1929.

16. Rosengren SM, Welgampola MS, Colebatch JG. Vestibular evoked myogenic potentials: past, present and future. Clin Neurophysiol. 2010;121(5):636-651.

17. Curthoys IS. A critical review of the neurophysiological evidence underlying clinical vestibular testing using sound, vibration and galvanic stimuli. Clin Neurophysiol. 2010;121(2):132-144.

18. Holmeslet B, Foss OA, Bugten V, Brantberg K. Ocular vestibular-evoked myogenic potentials (oVEMPs) in response to bone-conducted vertex vibration. Clin Neurophysiol. 2015;126(3):608-613.

19. Curthoys IS, Kim J, Mcphedran SK, Camp AJ. Bone conducted vibration selectively activates irregular primary otolithic vestibular neurons in the guinea pig. Exp Brain Res. 2006;175(2):256-267.

20. Papathanasiou ES, Murofushi T, Akin FW, Colebatch JG. International guidelines for the clinical application of cervical vestibular evoked myogenic potentials: an expert consensus report. Clin Neurophysiol. 2014;125(4):658-666.

21. Brantberg K, Löfqvist L, Westin M, Tribukait A. Skull tap induced vestibular evoked myogenic potentials: an ipsilateral vibration response and a bilateral head acceleration response? Clin Neurophysiol. 2008;119(10):2363-2369.

22. Westin M, Brantberg K. Mastoid and vertex low-frequency vibrationinduced oVEMP in relation to medially directed acceleration of the labyrinth. Clin Neurophysiol. 2014;125(3):615-620.
23. Krause E, Mayerhofer A, Gürkov R, et al. Effects of acoustic stimuli used for vestibular evoked myogenic potential studies on the cochlear function. Otol Neurotol. 2013;34(7):1186-1192.

24. Mattingly JK, Portnuff CD, Hondorp BM, Cass SP. Sudden Bilateral Hearing Loss After Cervical and Ocular Vestibular Evoked Myogenic Potentials. Otol Neurotol. 2015;36(6):961-964.

25. Deepak DT, Bhat JS, Kumar K. Ocular vestibular evoked myogenic potential using different test stimuli. ISRN Otolaryngol. 2013;2013:161937.

26. Plontke S, Zenner HP. Current aspects of hearing loss from occupational and leisure noise. GMS Curr Top Otorhinolaryngol Head Neck Surg. Epub 2004 Dec 28.

27. Welgampola MS, Rosengren SM, Halmagyi GM, Colebatch JG. Vestibular activation by bone conducted sound. J Neurol Neurosurg Psychiatry. 2003;74(6):771-778.

28. Rosengren SM, Colebatch JG, Straumann D, Weber KP. Single motor unit responses underlying cervical vestibular evoked myogenic potentials produced by bone-conducted stimuli. Clin Neurophysiol. 2015;126(6):1234-1245.

29. Iwasaki S, Mcgarvie LA, Halmagyi GM, et al. Head taps evoke a crossed vestibulo-ocular reflex. Neurology. 2007;68(15):227-1229.

30. Dennis DL, Govender S, Colebatch JG. Properties of cervical and ocular vestibular evoked myogenic potentials (cVEMPs and oVEMPs) evoked by $500 \mathrm{~Hz}$ and $100 \mathrm{~Hz}$ bone vibration at the mastoid. Clin Neurophysiol. 2016;127(1):848-857.

31. Verrecchia L, Westin M, Duan M, Brantberg K. Ocular vestibular evoked myogenic potentials to vertex low frequency vibration as a diagnostic test for superior canal dehiscence. Clin Neurophysiol. 2016;127(4):2134-2139.

32. Kantner C, Gürkov R. The effects of commonly used upward gaze angles on ocular vestibular evoked myogenic potentials. Otol Neurotol. 2014;35(2):289-293.

33. Katz J. Handbook of Clinical Audiology. $5^{\text {th }}$ ed. Philadelphia: Lippincott Williams \& Wilkins; 2002.

34. Stenfelt S. Transcranial attenuation of bone-conducted sound when stimulation is at the mastoid and at the bone conduction hearing aid position. Otol Neurotol. 2012;33(2):105-114.

35. Reinfeldt $\mathrm{S}$, Stenfelt $\mathrm{S}$, Håkansson B. Estimation of bone conduction skull transmission by hearing thresholds and ear-canal sound pressure. Hear Res. 2013;299:19-28.

36. von Békésy G. Experiments in Hearing. Oxford University Press: New York; 1960.

37. Håkansson B, Carlsson P, Tjellström A. The mechanical point impedance of the human head, with and without skin penetration. J Acoust Soc Am. 1986;80(4):1065-1075.
Medical Devices: Evidence and Research

\section{Publish your work in this journal}

Medical Devices: Evidence and Research is an international, peerreviewed, open access journal that focuses on the evidence, technology, research, and expert opinion supporting the use and application of medical devices in the diagnosis, monitoring, treatment and management of clinical conditions and physiological processes. The identification of novel
Dovepress

devices and optimal use of existing devices which will lead to improved clinical outcomes and more effective patient management and safety is a key feature. The manuscript management system is completely online and includes a quick and fair peer-review system. Visit http://www. dovepress.com/testimonials.php to read real quotes from authors. 